\title{
Inadvertent Puncture Of Right Vertebral Artery During Central Venous Line Catheter Insertion
}

Mohd Nabil Halim ${ }^{1}$, Azrina Md Ralib ${ }^{1}$, Suhaila Md Nayan ${ }^{1}$, Muhammad Rasydan Abdul Ghani ${ }^{1}$, Nur Fariza Ramly ${ }^{1}$, Nurfatihah Alias ${ }^{1}$

${ }^{1}$ Department of Anaesthsiology and Intensive Care, Kulliyyah of Medicine, International Islamic University of Malaysia

Presenter: Mohd Nabil Halim

The use of ultrasound has been proposed to reduce the number of complications and to increase the safety and quality of central venous catheter (CVC) placement. In this report, we recommend the structures approach for US-guided to insert venous access for clinical practice. To achieve the best skill for CVC placement, the knowledge from anatomic landmark techniques and knowledge from US-guided CVC placement need to be combined and integrated. 59 years old lady, planned for total abdominal hysterectomy for pelvic tumour excision. Anticipate massive bleeding with major fluids shift during intraoperative, invasive monitoring with CVC was inserted preoperative at right internal jugular vein. The procedure of insertion was done using US-guided with out-of-plane method. While connected to CVP monitoring noted to have arterial wave. Decided to keep the central venous line in-situ and referral to radiologist for imaging was arranged. CT angiogram's findings of a catheter were seen to transverse the right internal jugular vein through-and-through and seen to lie within the right vertebral artery coursing into the right subclavian artery. No evidence of carotid artery injury. Referred to intervention radiology and vascular surgery team for the best method of removal the central line catheter. Removal of CVC for inadvertent injury to right verterbral artery under guided contrast study by intervention radiologist. The case illustrates the importance of ultrasound-guided CVL insertion. 